Proceedings of Anticancer Research

Research Article

\title{
Clinical Outcomes of Artificial Total Hip Arthroplasty for Proximal Femoral Bone Tumors
}

\author{
Miao Ma ${ }^{1}$, Yajun Wang ${ }^{2}$, Guifu $\mathrm{Ma}^{3}$,Wenyuan $\mathrm{Luo}^{3}$, Yaowen Qian ${ }^{3}$ \\ ${ }^{1}$ Gansu University of Chinese Medicine, Zhangye 734000, Gansu Province, China; \\ ${ }^{2}$ Zhang Ye People's Hospital affiliated to Hexi University, Lanzhou 730000, Gansu Province, China; \\ ${ }^{3}$ Gansu Provincial People’s Hospital, Lanzhou 730000, Gansu Province, China
}

\begin{abstract}
Objective: To investigate the clinical outcome of artificial hip replacement for proximal femoral bone tumors. Methods: One hundred patients with proximal femur bone tumors admitted to the hospital for treatment from June 2018 to May 2020 were selected and divided into a control group and an experimental group of 50 patients each using central randomization. The control group received conventional treatment and the experimental group underwent artificial total hip arthroplasty, and the results of treatment were compared between the two groups. Results: The operative time and hospitalization time of the experimental group were shorter than that of the control group, and the blood loss was less than that of the control group; after the replacement surgery, the range of flexion and extension, internal and external rotation activity and abduction activity scores were better than those of the control group; and the total incidence of adverse reactions in the experimental group was $6.0 \%$, which was significantly lower than that of the control group $(14.0 \%)$. By comparing the treatment effects of the two groups, the differentiation was significant, and $\mathrm{P}<0.05$ was statistically significant. Conclusion: Artificial hip arthroplasty can effectively treat proximal femoral tumors, shorten operative time, hospitalization time and intraoperative bleeding, and alleviate patients' therapeutic pain, which has good promotion value in clinical practice.
\end{abstract}

Keywords: Total hip arthroplasty; Proximal femoral bone tumor; Clinical outcome

Publication date: September, 2020

Publication online: 30 September, 2020

*Corresponding author: Miao Ma, 943319401@ qq.com
Bone tumors are tumors that originate from the bones or their adnexal tissues. The proximal femur is the site of greatest stress concentration in the body, supporting most of the body weight under vertical and shear stresses, and the anatomy of the proximal femur varies greatly in individualization because of the peculiarities of blood supply, anatomical structure, and biomechanics, and the proximal femur has become a common site of limb preservation in the occurrence of bone tumors $^{[1]}$. When these bone tissues in the proximal femur develop neoplastic lesions, they will reduce the strength of the bone tissue and will be prone to pathological fractures, which can cause pain, swelling, and other symptoms and dysfunctions that can seriously affect the patient's normal life and quality of life ${ }^{[2]}$. Following an attack, patients may experience pain, swelling or lumps, dysfunction and other symptoms which can have a particular impact on their physical functioning. According to recent relevant medical literature, surgical treatment of proximal femoral bone tumors is more common and it has excellent clinical outcomes, and limb-sparing surgery has gradually gained recognition among clinicians ${ }^{[3]}$. Among them, tumor-type prosthesis can restore joint function faster, with a lower incidence of early complications and better limb function after surgery, which has become the most widely used reconstruction method in current limb-sparing surgery ${ }^{[4]}$. In order to further understand the role of artificial hip arthroplasty in the treatment of proximal femoral tumors, 100 patients with proximal femoral tumors in our hospital were selected and treated in groups in this study.

\section{Information and methods}




\subsection{Basic information}

In this study, 100 patients with tumors of the proximal femur who were admitted to our hospital for treatment from June 2018 to May 2020 were divided into 50 patients each in the control and experimental groups by central randomization. There were 25 males and 25 females in the control group, aged 13-69 years (42.3 \pm 7.6 years). There were 43 cases of benign tumors and neoplastic lesions (11 cases of fibroplasia, 7 cases of aneurysmal bone cysts, 7 cases of bone cysts, 2 cases of endogenous chondromas, 2 cases of osteochondrosis, 4 cases of benign fibroblastoma, 3 cases of osteoblastoma, 3 cases of chondroblastoma, 4 cases of giant cell tumor) and 7 cases of malignant tumors ( 2 cases of chondrosarcoma, 2 cases of osteosarcoma, 1 case of malignant fibrous tissue tumor, 2 cases of metastasis). There were 28 male and 22 female patients, aged $12-68$ years $(42.5 \pm 7.5$ years $)$ in the experimental group. There were 45 patients with benign tumors and neoplastic lesions (13 cases of fibrous structural hyperplasia, 9 cases of aneurysmal bone cysts, 5 cases of osteoblasts, 3 cases of endogenous chondromas and osteochondromas, 3 cases of benign fibroblastomas, 3 cases of osteoblastomas, 4 cases of chondroblastomas, and 5 cases of giant cell tumors) and 5 cases of malignant tumors ( 1 case of chondrosarcoma, 2 cases of osteosarcoma, and 2 cases of malignant fibrous tissue tumors).The difference in general information between the two groups of patients was not statistically significant and could be studied for comparison. Inclusion criteria: (i) The diagnostic criteria of bone tumor of the proximal femur were met. (ii) Patients agreed to this study. Exclusion criteria: (i) non-surgical patients; (ii) symptoms that did not meet the diagnostic criteria for bone tumors of the proximal femur.

\subsection{Treatment methods}

Patients in the control group received routine surgical treatment. After hospitalization, patients were systematically examined to obtain detailed information about their diseases and to formulate a surgical plan based on the results of the examination.If the patient has minimal bone destruction, the location of the lesion can be scraped directly. If the lesion area is too large, the curettage is added to the scraping to inactivate the tumor wall.

Patients in the experimental group underwent total hip arthroplasty with the following details: before treatment, patients underwent systematic examination to determine whether they were suitable for artificial hip joint surgery; after meeting the conditions, general anesthesia was administered to the patients, then the patients were placed in the supine position, the hip joint was placed in the movable position of the operating bed, the tip of the greater trochanter was centered, the end of the femur was exposed, a 7-10 cm incision was made in the anterior superior skeletal spine, the vastus lateralis muscle fascia was cut, the lateral femoral artery was explored, the lateral femoral muscles were exposed, and the pull hooks were placed on the medial and lateral femoral neck, at the greater trochanter and the anterior wall of the acetabulum, and the medial and lateral muscles were pulled. In a clockwise direction, the joint capsule was incised, parallel to the osteotomy, and the osteotomy block and femoral head were removed separately. After completing the osteotomy, we removed the lateral pulling hooks, removed the periacetabular osteophytes, tested the mold according to the direction of the predetermined mortar cup, then internally and externally rotated the affected hip joint. Subsequently, the femur was exposed. The femoral prosthesis was installed. Then we took a curette to probe the medullary cavity and expanded the medulla to determine the mobility and stability, and then placed the prosthetic femoral head and reseted.

\subsection{Evaluation of indexes}

Intraoperative time, blood loss and length of hospital stay were observed and the overall incidence of flexion, internal/external rotation and abduction mobility and adverse events were compared between the two groups.

(i) Flexion, internal/external rotation, and abduction mobility. The Harris Hip Functional Assessment Criteria (percentile) are used for assessment, and the higher the patient score, the better the outcome.

(ii) Adverse effects. After treatment, mainly bone metastases, minor infections and anemia were recorded.

\subsection{Data statistics}

The study data were analyzed statistically using SPSS 20.0 software.Using the independent samples t-test, the resulting measurements were expressed as $\left(\mathrm{x}^{-} \pm \mathrm{s}\right)$ and the coefficient data were expressed as percentages (\%) using the $\mathrm{x} 2$ test or Fisher's exact probability method. $\mathrm{P}<0.05$ was statistically significant.

\section{Results}

\subsection{Comparison of operative time, blood loss and hospital stay between the two groups of patients}

The results showed that the experimental group had 
Table 1. Statistics of operative time, blood loss and length of hospital stay during treatment in the two groups of patients

\begin{tabular}{|c|c|c|c|}
\hline Group & Operating time $(\mathrm{min})$ & hemorrhage $(\mathrm{ml})$ & length of hospital stay (d) \\
\hline Experimental group $(\mathrm{n}=50)$ & $99.8 \pm 13.6^{*}$ & $849.5 \pm 30.6^{*}$ & $17.5 \pm 5.8^{*}$ \\
\hline Control group $(n=50)$ & $120.2 \pm 26.4$ & $985.5 \pm 50.9$ & $25.4 \pm 7.8$ \\
\hline
\end{tabular}

Compared with the control group, $* \mathrm{P}<0.05$

Table 2. Statistics of scores for the evaluation of flexion, external and internal rotation and abduction mobility in the two groups of patients

\begin{tabular}{|c|c|c|c|}
\hline Group & Flexural mobility & $\begin{array}{l}\text { Internal and external rotation } \\
\text { mobility }\end{array}$ & Degree of outreach \\
\hline Experimental group $(\mathrm{n}=50)$ & $48.5 \pm 7.2 *$ & $17.7 \pm 4.3 *$ & $18.5 \pm 4.4 *$ \\
\hline Control group $(n=50)$ & $34.5 \pm 6.2$ & $12.4 \pm 3.3$ & $12.5 \pm 4.7$ \\
\hline
\end{tabular}

Compared with the control group, ${ }^{*} \mathrm{P}<0.05$

Table 3. Statistics on the overall incidence of postoperative adverse events in the two groups of patients

\begin{tabular}{|c|c|c|c|c|}
\hline Group & Skeletal metastases & Slight infection & Anemic & Totals \\
\hline Experimental group $(n=50)$ & $1(2.0)$ & $1(2.0)$ & $1(2.0)$ & $3(6.0) *$ \\
\hline Control group $(\mathrm{n}=50)$ & $3(6.0)$ & $2(4.0)$ & $2(4.0)$ & $7(24.0)$ \\
\hline
\end{tabular}

Compared with the control group, ${ }^{*} \mathrm{P}<0.05$

shorter intraoperative time and hospital stay than the control group, and the intraoperative blood loss was significantly lower than that of the control group. $\mathrm{P}<0.05$ was statistically significant. (Table 1 ).

\subsection{Comparison of postoperative flexion, internal/ external rotation and abduction mobility between the two groups of patients}

After comparison, the scores of the three activity indexes evaluated in the postoperative experimental group were higher than those in the control group, and $\mathrm{P}<0.05$ was statistically significant (see Table 2 for details).

\subsection{Comparison of the overall incidence of adverse events between the two groups after surgery}

A comparison of the two groups showed that some adverse effects existed after surgical treatment. The overall adverse birth rate in the experimental group was only 3 cases $(6.0 \%)$, which was significantly lower than the 7 cases $(24.0 \%)$ in the control group, with a statistically significant difference of $\mathrm{P}<0.05$ between the groups ( Table 3).

\section{Discussion}

The proximal femur is an important weight-bearing bone in the body and is one of the most common areas for skeletal tumors. Tumors of the proximal femur cause pain, swelling, and dysfunction and, when pathological fractures occur, patients experience increased pain and loss of mobility, thereby reducing quality and duration of life ${ }^{[5]}$.

Over the years, with the continuous improvement of medical care in China, surgical resection of diseased bone is an effective treatment for malignant tumors of the proximal femur. Total hip arthroplasty has attracted widespread attention in the treatment of proximal femoral tumors. When tumorigenic lesions occur in the proximal femur, it often causes significant pain. The commonly-used bone reconstruction methods after resection of proximal femur tumor include allogeneic bone reconstruction, artificial joint-allogeneic bone composite reconstruction and tumor-type prosthesis reconstruction. Tumor-based prosthesis reconstruction has the following advantages over other reconstruction methods: (1) Simple operation and short operative time; (2) Low complication rates such as infection, bone discontinuity, and rejection reaction; (3) Early functional exercise can be performed to shorten recovery time; (4) Good postoperative limb function ${ }^{[6]}$.

Total hip arthroplasty has been clinically shown to allow patients to walk with weight bearing as soon as possible after surgery, to help restore lower extremity 
and hip function, and to more completely remove tumors to reduce recurrence and maintain joint function.

In this study, through the comparative analysis of the two groups of patients in the intraoperative indicators, the results show that the experimental group of patients in the intraoperative time, hospital stay, bleeding and the total incidence of adverse events are lower than the control group, at the same time, the postoperative evaluation score of the activity indicators of the patients in the experimental group is also significantly higher than that of the control group, obviously, $\mathrm{P}<0.05$ is statistically significant. However, there are still some shortcomings in this study, for example, factors associated with postoperative local recurrence have not been found, so cases need to be collected for further study. In order to improve the safe and effective surgical procedures for patients with bone tumors, it is important to improve the recovery of postoperative limb function and the overall favorable prognosis of patients.

\section{References}

[1] Philippon MJ, Michalski MP,Campbell KJ,et al.Surgically relevant bony and soft tissue anatomy of the proximal femur[J]. Orthop JSports Med, 2014, 2(6): 2325967114535188.

[2] Pu FF, Zhong BL, Liu JX, et al. Cement-based artificial bipolar femoral head replacement for proximal femoral bone metastases[J]. China Cancer Clin, 2020, 47(10): 501-506.

[3] Guzik G. Oncological and functional results after surgical treatment of bone metastases at the proximal femur[J]. BMC Surg, 2018, 18(1): 5.

[4] Ji JT, Zhang H, Hu YC, et al. Preliminary application of bone stem prosthesis in the reconstruction of bone defects after resection of proximal femur malignant tumor[J]. Chinese Journal of Orthopaedics, 2015, 35(3): 203-211.

[5] Chen F, Pu F. Safety of denosumab versus zoledronic acid in pa-tients with bone metastases: a meta-analysis of randomized con-trolled trials[J]. Oncol Res Treat, 2016, 39(7-8): 453-459.

[6] Stevenson JD,Kumar VS,Cribb GL,et al.Hemiarthroplasty proximal femoral endoprostheses following tumour reconstruction:is ace-tabular replacement necessary[J]. Bone Joint J, 2018, 100-B(1): 101-108. 